\title{
Haptic concepts in the blind
}

\author{
Donald Homa, Kanav Kahol, Priyamuada Tripathi, \\ Laura Bratton, and Sethuraman Panchanathan \\ Arizona State University, Tempe, Arizona
}

\begin{abstract}
We investigated and compared the acquisition of haptic concepts by the blind with the acquisition of haptic concepts by sighted controls. Each subject — blind, sighted but blindfolded, sighted and touching, and sighted only-initially classified eight objects into two categories using a study/test format, followed by a recognition/ classification test involving old, new, and prototype forms. Each object varied along the dimensions of shape, size, and texture, with each dimension having five values. The categories were linearly separable in three dimensions, but no single dimension permitted $100 \%$ accurate classification. The results revealed that blind subjects learned the categories quickly and comparably with sighted controls. On the classification test, all groups performed equivalently, with the category prototype classified more accurately than the old or new stimuli. The blind subjects differed from the other subjects on the recognition test in two ways: They were least likely to false alarm to novel patterns that belonged to the category but most likely to false alarm to the category prototype, which they falsely called "old" $100 \%$ of the time. We discuss these results in terms of current views of categorization.
\end{abstract}

Research in formal categorization theory has made extensive contributions to our knowledge of human memory and perception. However, formal categorization theory has evolved primarily from studies that have used stimuli that are presented visually. A handful of studies have explored auditory (Aiken, 1969; Rosser, 1967) and kinesthetic concepts. For example, Solso and Raynis (1979) had subjects learn kinesthetic concepts in which arm motions were manipulated to trace out line figures.

Notably lacking in the domain of human categorization is how sighted individuals integrate haptic information into concepts and, more specifically, how individuals who are blind are able to learn object concepts. Haptics, derived from the Greek word haptikos, refers to the sense of touch, and includes all felt sensations derived from contact with texture, pressure, hardness, shape contours, temperature, and so on. A moment's reflection makes it clear that commonplace concepts are acquired from a confluence of modalities, with haptics playing an unknown but likely substantial role. The stroking of fur, the felt conformations of a face, exploring everyday objects, and the carrying of an infant or a small pet all involve commonplace haptic sensations. Early experiences are dominated by haptic influences. For example, infants are more likely to bang hard than soft objects (Lockman \& Wright, 1988) and to stroke or finger surfaces on the basis of whether they are smooth or textured (Ruff, 1984). In sighted individuals, identification of commonplace objects is remarkably accurate (Klatzky, Lederman, \& Metzger, 1985), and blind and sighted children do not differ in tactile exploratory strategies (Morrongiello, Humphrey, Timney, Choi, \&
Rocca, 1994). For the blind, haptic feedback is a primary mode of identification, and knowledge representation may be inherently dependent on perception through the haptic modality. Thus, formation of haptic concepts through the haptic modality is an important yet unexplored research direction.

The present study was an exploratory venture into how blind individuals learn and represent concepts when instances are explored through touch alone, contrasting their performance with subjects who were sighted but blindfolded. Two additional control groups were also used: subjects who were allowed to touch and see the objects, and subjects who were allowed to see but not touch the objects. The contrast in performance between sighted but blindfolded subjects and the sighted and touching subjects should tell us what sight adds to the haptic modality in learning concepts; the contrast between sight alone and touch alone provides an assessment of which modality alone better teaches the categories used in the present study.

Since the blind have been shown to perform worse in the identification of two- and three-dimensional objects than sighted but blindfolded subjects (Shimizu, Saida, \& Shimura, 1993), one hypothesis is that the blind would learn haptic concepts less readily than sighted but blindfolded subjects. However, other researchers have found that the blind were superior to sighted subjects in tasks involving tactile texture discrimination (Van Boven, Hamilton, Kauffman, Keenan, \& Pascual-Leone, 2000). Therefore, we were uncertain whether the blind subjects in our study would fare worse or better than the sighted

D. Homa, donhoma@asu.edu 


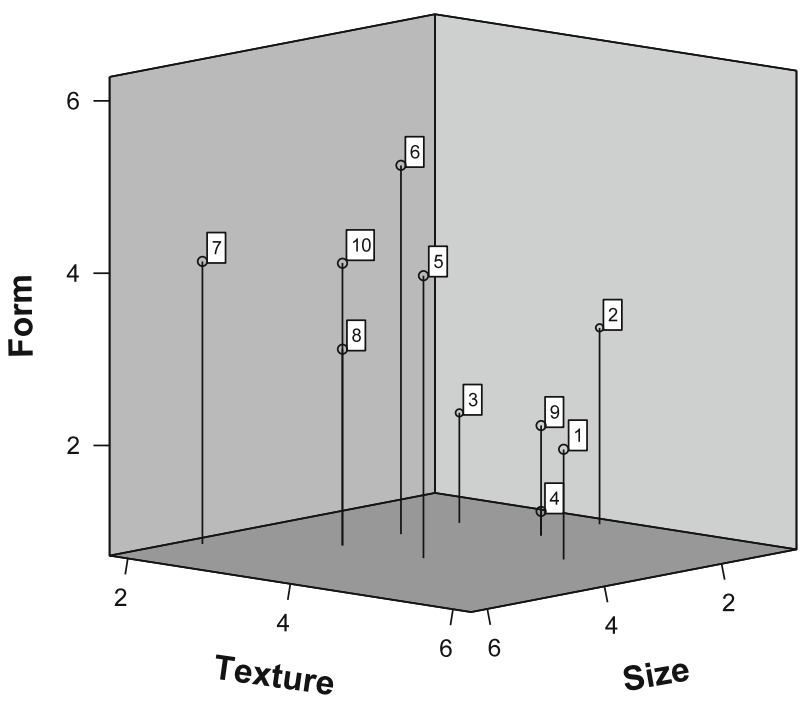

Figure 1. Three-dimensional representation of learning patterns (1-8) and category prototypes $(9,10)$. Category $A=\{1,2,3$, $4,9\}$; Category $B=\{5,6,7,8,10\}$.

controls. We were also interested in whether the blind subjects would be more likely to abstract the prototype for the learned categories, and whether reliance on exemplars or prototypes would increase or diminish with multimodal as opposed to single-modal input. The present work attempts to provide initial evidence on both issues.

To ensure that category learning was challenging for our subjects, we constructed a category space, shown in Figure 1, that mirrored the structure of a category space often used (Medin \& Schaffer, 1978; Smith \& Minda, 2000) that has proven to be difficult for sighted subjects and visual presentation. All objects varied along three dimensions - size, shape, and texture - with each dimension having five values. The learning objects belonged to either of two categories, with each category represented by four objects each. The category prototypes, presented only during the transfer test, had the modal values of their respective categories. ${ }^{1}$ The learning categories were separable by a linear discriminate function, but no single dimension was sufficient to separate the two categoriesthat is, perfect separation of the two categories required attention to at least two of the three dimensions.

We reasoned that this structure would be difficult to learn, since a similar structure used by Medin and Schaffer (1978), and replicated numerous times (Smith \& Minda, 2000), proved difficult to learn for sighted subjects. In the study by Medin and Schaffer (1978), eight binary-valued stimuli, four in each of two categories, formed the learning set, with each stimulus defined by a value on each of four dimensions; the remaining eight patterns formed the transfer set. This, and similar structures, were difficult to learn, with $40 \%-55 \%$ of the subjects failing to reach the errorless learning criterion after 16 (Experiment 2) or 32 (Experiment 3) learning blocks.

A couple of contrasts between our structure and the structure used by Medin and Schaffer (1978) and in related studies are worth noting. Medin and Schaffer used patterns that varied along four dimensions, whereas our patterns varied along three. However, each of the patterns in Medin and Schaffer were binary valued on each dimension, generating a population of 16 stimuli. Since our objects could take on any of five values on each dimension, the resulting population contained 125 different objects. In neither the current study nor in Medin and Schaffer's experiment was it possible to use a single dimension to achieve errorless learning performance. As a consequence, we anticipated that the learning of the category structure used in the present study would be difficult.

We were also interested in how the blind and sighted control subjects would perform on a transfer test involving both the classification of new objects as well as their ability to recognize old (training) from new objects. The training and transfer objects are shown, schematically, in Table 1. Typically, sighted subjects show an advantage in the classification of old to new stimuli on a transfer test, with classification of the prototype intermediate or even exceeding the level of accuracy of the old stimuli, at least on an immediate test (e.g., Homa, Goldhardt, BurruelHoma, \& Smith, 1993; Posner \& Keele, 1968; Solso \& Raynis, 1979). We hypothesized that our blind subjects would show no disadvantage, relative to the sighted controls, in the classification of new and prototypical objects, given that terminal levels of learning matched those of the sighted controls.

The recognition judgment provided a potential test of whether the blind subjects would be most likely of the four groups to abstract a haptic prototype. False recognition of the category prototype has been obtained repeatedly and has been interpreted as support for an abstraction mechanism (Homa et al., 1993; Metcalfe \& Fisher, 1986; Solso

Table 1

Schematic Representation of the Two Categories in Learning

\begin{tabular}{|c|c|c|c|c|}
\hline \multirow[b]{2}{*}{ Task } & \multirow[b]{2}{*}{ Stimulus } & \multicolumn{3}{|c|}{ Dimension } \\
\hline & & Shape & Texture & Size \\
\hline \multirow[t]{4}{*}{ Training A } & Form 1 & 2 & 5 & 3 \\
\hline & Form 2 & 3 & 4 & 1 \\
\hline & Form 3 & 2 & 3 & 2 \\
\hline & Form 4 & 1 & 4 & 2 \\
\hline \multirow[t]{6}{*}{ Training B } & Form 5 & 4 & 4 & 4 \\
\hline & Form 6 & 5 & 3 & 3 \\
\hline & Form 7 & 4 & 2 & 5 \\
\hline & Form 8 & 3 & 3 & 4 \\
\hline & Proto A & 2 & 4 & 2 \\
\hline & Proto B & 4 & 3 & 4 \\
\hline \multirow[t]{5}{*}{ Transfer-New A } & Form 9 & 2 & 1 & 4 \\
\hline & Form 10 & 1 & 3 & 1 \\
\hline & Form 11 & 1 & 5 & 3 \\
\hline & Form 12 & 2 & 2 & 5 \\
\hline & Form 13 & 3 & 5 & 2 \\
\hline \multirow[t]{5}{*}{ Transfer-New B } & Form 14 & 5 & 5 & 2 \\
\hline & Form 15 & 5 & 1 & 3 \\
\hline & Form 16 & 3 & 1 & 3 \\
\hline & Form 17 & 4 & 1 & 3 \\
\hline & Form 18 & 4 & 5 & 2 \\
\hline
\end{tabular}

Note-Increasing ordinal values for size represent increasing size; for texture, increasing roughness. For the shape dimension, the ordinal values are arbitrary. 
\& Raynis, 1979). Because the blind exhibit superior texture discrimination in comparison with sighted subjects (Van Boven et al., 2000), the blind subjects might avoid false recognition of the category prototype. This might be expected if the blind are more likely to store distinctive haptic codes for the training objects. If, however, the blind subjects classify the category prototype with high accuracy while exhibiting a high false alarm rate to the category prototype, then the hypothesis that the blind subjects abstracted a haptic prototype would be supported (although caution regarding this interpretation is noted in the Discussion).

The recognition task also permitted a test of a hypothesis provided informally by blind students: Blind subjects should be more conservative than their sighted controls. A number of blind students gave anecdotal accounts of how they had to be more cautious than the sighted, since a false alarm (such as the taking of a wrong medication) could have disastrous consequences. As a result, we hypothesized that our blind subjects would show a lower false alarm, and perhaps a lower hit rate, than the sighted controls. Since the results were amenable to signal detection analysis (Green \& Swets, 1966), this would be revealed by the placement of a more conservative criterion, separate from their ability to discriminate old from new $\left(d^{\prime}\right)$.

In learning, a multiple study/test procedure was used. On each trial, all learning objects were presented, singly and randomly, to the subject, with correct identification (e.g., "This is an A"). Following the presentation of all eight training objects, each object was randomly selected and presented to the subject for classification. Following his or her response, correct feedback was again provided. This study/test procedure was used four times in all and constituted the learning phase.

Following learning, a transfer test was given in which old, new, and prototype objects from each category were presented in a random order. The subject was required to make two judgments: first to indicate whether the object was old or new, and then to classify the object into Category A or Category B, as in learning. Instructions emphasized that an object should be called "old" only if it was identical to one of the objects presented in the learning phase. No feedback was provided during the transfer test.

\section{METHOD}

\section{Subjects}

The subjects were 83 students at Arizona State University: 20 blind students and 63 sighted students, with approximately equal numbers randomly assigned to the three control conditions (sighted but blindfolded, sighted and touching, sighted only). The individuals who were blind could be grouped into early, mid-, and late blind, based on the age at which they lost their sight. ${ }^{2}$ The 8 early blind subjects included 5 individuals who were congenitally blind and 3 who lost their visual abilities before the age of 3 . The 6 mid-blind subjects lost their vision between 3 and 9 years of age, and the 6 late blind lost their sight after the age of 12. All of the blind subjects lost their sight due to diseases that affected the peripheral components of the visual system and otherwise exhibited no additional neurological problems. The average age of the subjects who were blind was 35.2 years, with a spread of 15.1 years. Sixteen of the blind subjects

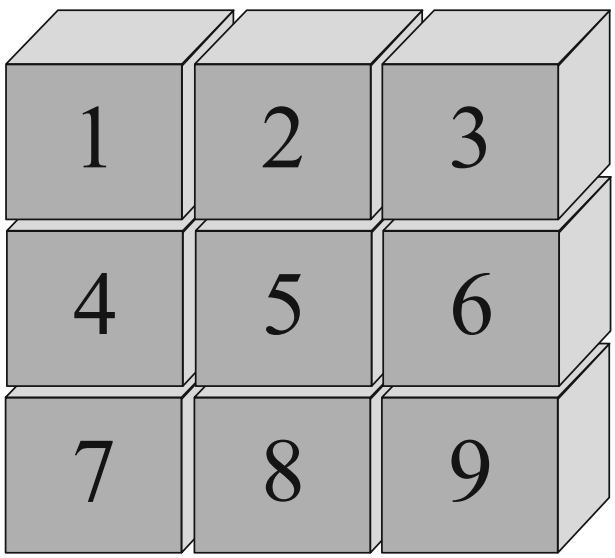

Figure 2. The nine-block grid.

had no vision, 2 had light vision, and 2 could read large print. The individuals with light vision could discriminate light from dark and could detect movements but had, otherwise, no functional vision. The 2 individuals who could read large print were legally blind and could not navigate their environment from sight alone. Nonetheless, these 2 subjects were blindfolded during the learning and test trials. The sighted, blindfolded group had an average age of 22.3 years, with a spread of 4.2 years. The sighted, touching group had an average age of 23.2 years, with a spread of 3.4 years. The sighted-only group had an average age of 22.8 years, with a spread of 4.1 years. All the sighted users had vision correctable to $20 / 20$.

\section{Materials}

All objects were derived from a basic nine-block pattern, as depicted in Figure 2. All learning objects in Category A had the middle block removed (Block 5) and tended to be smaller in size, with a rougher texture than the objects in Category B had. The Category B objects had a middle notch removed from the top or bottom (Block 2 or 8) and tended to be larger in size, with a smoother texture than the Category A objects had. No dimension by itself was sufficient for classification. For example, one Category B object had a center block removed, one Category A object matched a Category B object in size, and three Category B objects matched Category A objects for texture. The two categories, however, were separable in two dimensions; for example, considering only the size and texture dimensions, all Category A objects were linearly separable from the Category B objects (regardless of their shape). The five basic shapes used in the present experiment are shown in Figure 3.

Texture variations were controlled by covering the surface of a form with different grades of sandpaper. Size variations were created by varying the size of the blocks. The specific sizes and textures (sandpaper grits) are shown in Table 2.

The transfer stimuli included the 8 training objects, the two category prototypes, and 10 additional new objects (noted in the bottom half of Table 1). The 5 new objects belonging to Category A similarly had the center block removed, and none of the Category B objects had this cell removed. Also, as was the case with the learning objects, the new Category A objects tended to be larger ( 3 of the 5 patterns) and of smoother texture ( 3 of the 5 objects). These new patterns occupied regions on either side of the linear discriminate function that separated the two training categories and, therefore, could be assigned correctly to Categories A or B. ${ }^{3}$

\section{Procedure}

Before the experiment, the subjects were informed that the learning stimuli belonged to two separate categories (A and B) and that the objects varied in size, shape, and texture. The experiment was divided into two phases. In the learning phase, subjects were presented 


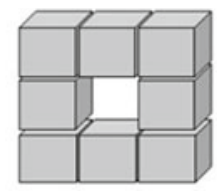

1

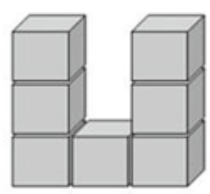

2

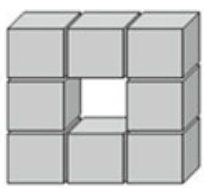

3

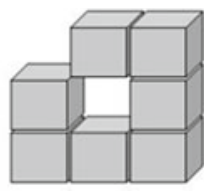

4

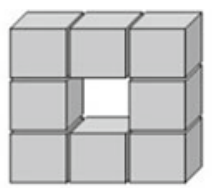

A

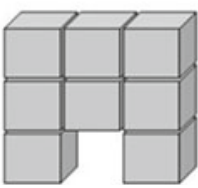

5

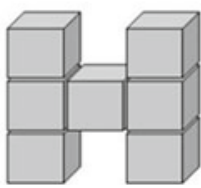

6

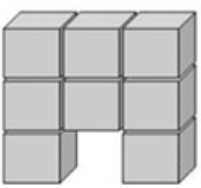

7

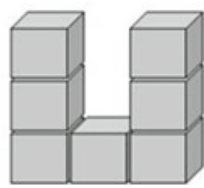

8

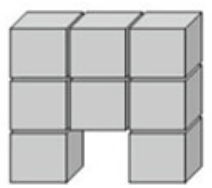

B

Figure 3. Training patterns (Forms 1-8) and Prototypes A and B.

with Objects $1-8$, in a random order, and were informed of their category. No restrictions were imposed on exploration strategy by the subjects. In haptic exploration, the subjects could use both hands for exploration, or one hand, and they could rotate and explore the stimuli. With visual exploration, the subjects were allowed to freely inspect the stimuli for as long as they desired. Following inspection of all eight objects, the objects were then presented in a random order, and the subjects were asked to identify the category (A or B) of each object. Following their response, corrective feedback was provided ("No, this is a B"). This study/test format was repeated four times and constituted the learning phase.

Following learning, all subjects received a transfer test in which old objects (Forms 1-8) and new objects (Prototype A, Prototype B, and the new stimuli) were presented. Of the 10 new objects, 5 were closer to A and 5 were closer to B. The subject was randomly presented an object and asked to identify whether this object was old or new and to determine its category membership. No feedback for either the recognition or classification judgment was provided. The entire transfer phase was, again, self-paced.

\section{RESULTS}

\section{Learning}

Figure 4 shows the mean learning rate across the four learning blocks, shown separately for the blind (B), sighted but blindfolded (S-BF), sighted-only (S), and sighted and touching (ST) subjects.

Each of the four groups learned rapidly, with performance on the last trial approaching $100 \%$ correct performance. The main effect of learning blocks was significant $\left[F(3,237)=20.49, M S_{\mathrm{e}}=.401, p<.01\right]$, as was the effect of groups $\left[F(3,79)=3.20, M S_{\mathrm{e}}=.875, p<.05\right]$. The

Table 2

Texture and Size Variations of Learning and Transfer Patterns

\begin{tabular}{lcl}
\hline Feature & Level & Actual Value \\
\hline Texture 1 & 1 & 400 grit \\
Texture 2 & 2 & 220 grit \\
Texture 3 & 3 & 150 grit \\
Texture 4 & 4 & 100 grit \\
Texture 5 & 5 & 60 grit \\
Size 1 & 1 & 4 in. $\times 4$ in. \\
Size 2 & 2 & 6 in. $\times 6$ in. \\
Size 3 & 3 & 8 in. $\times 8$ in. \\
Size 4 & 4 & 10 in. $\times 10$ in. \\
Size 5 & 5 & 12 in. $\times 12$ in. \\
\hline
\end{tabular}

interaction between blocks and group was not significant $(F<1)$. Overall, the mean accuracy for the blind subjects was .956, which compared favorably with the sighted-only (.948) and the sighted and touching (.972) subjects; the sighted but blindfolded subjects had the lowest rate of learning (.917). An LSD post hoc test $(p<.05)$ revealed that $\mathrm{ST}>\mathrm{B}=\mathrm{S}>\mathrm{BF}$. Although significant learning across the trial blocks occurred, each group demonstrated

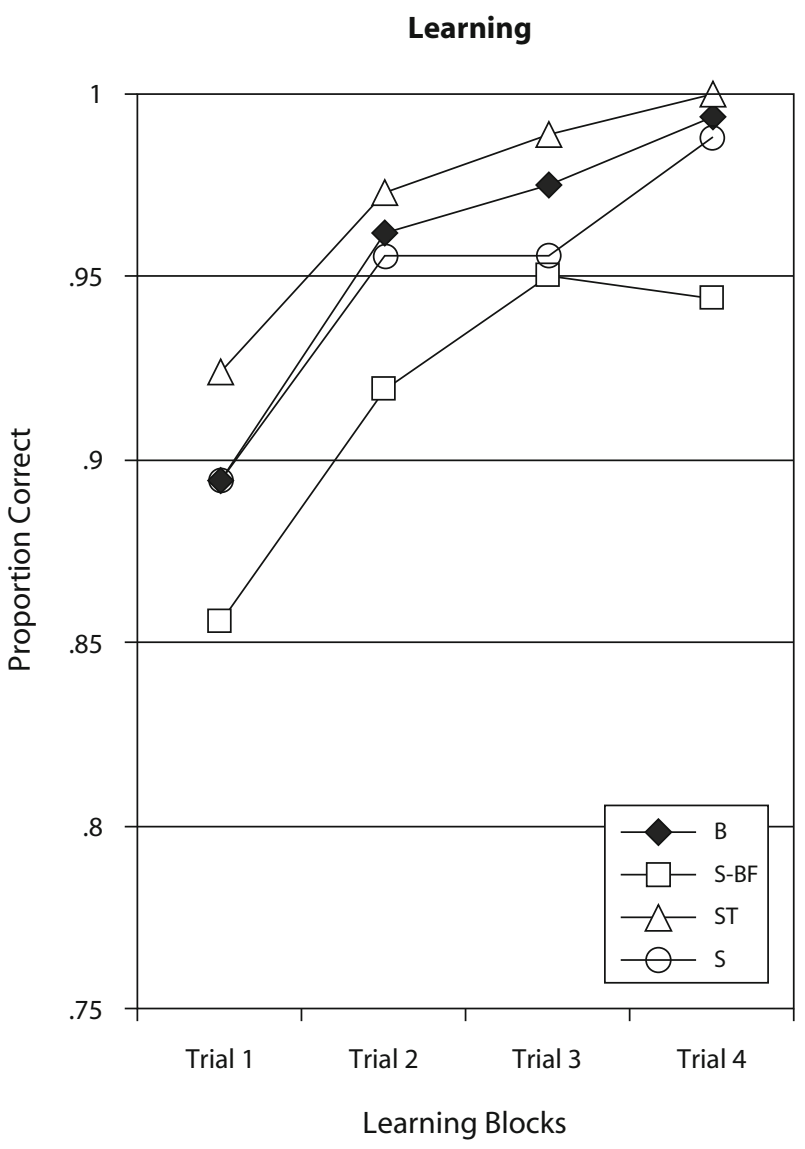

Figure 4. Proportion correct across learning blocks for the four groups (B, blind; S-BF, sighted but blindfolded; ST, sighted and touching; $\mathrm{S}$, sighted only). 


\section{Classification}

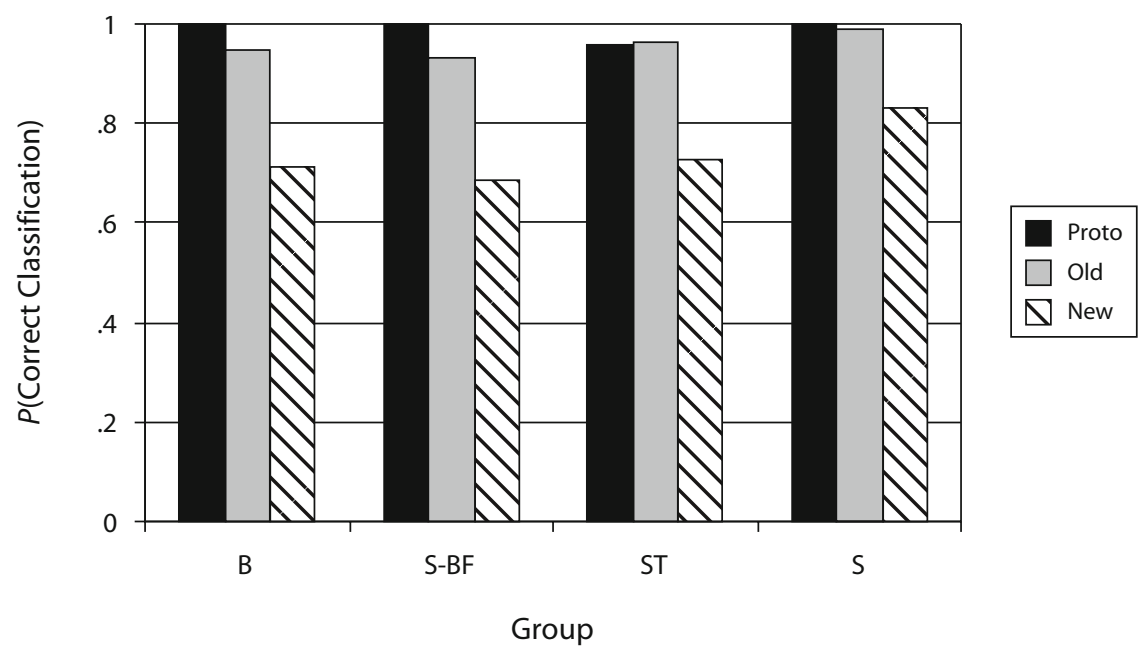

Recognition

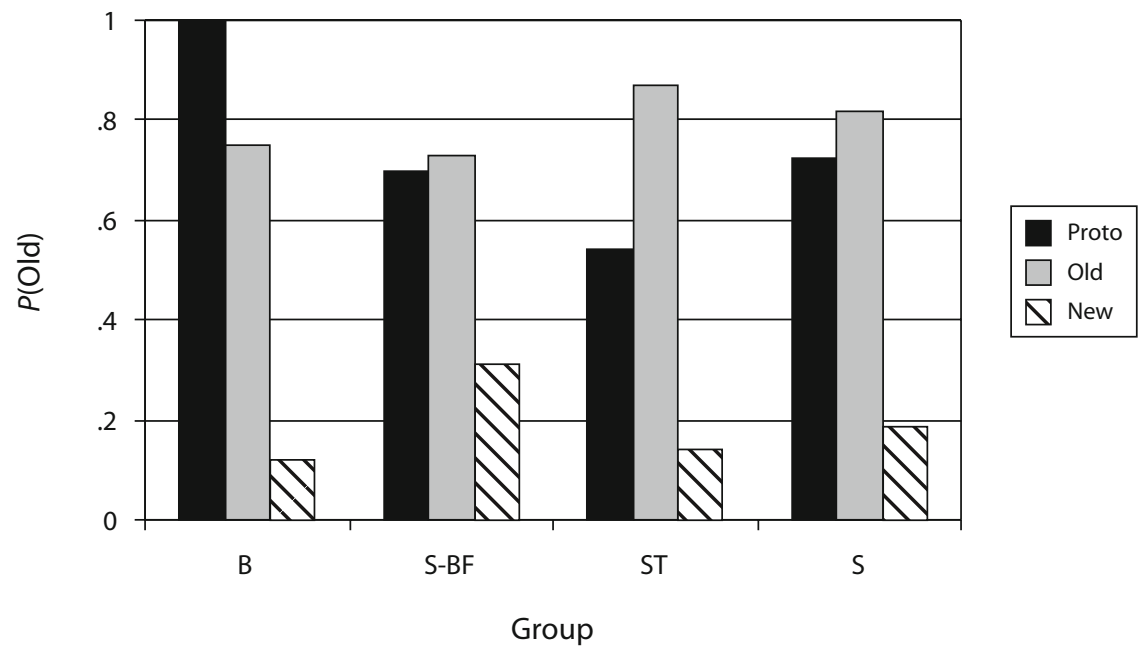

Figure 5. Transfer performance on old, prototype, and new patterns for four groups. The top half shows mean classification accuracy; the bottom half shows the likelihood of calling transfer patterns "old." B, blind; S-BF, sighted but blindfolded; ST, sighted and touching; S, sighted only.

fairly accurate learning after the initial study trial, with only the S-BF performing at less than $90 \%(.856)$.

\section{Transfer}

Figure 5 shows the classification (top panel) and recognition (bottom panel) of the old, new, and prototype objects. Overall, subjects classified the prototype objects (.989) and old objects (.958) more accurately than the new objects $(.737)\left[F(2,158)=103.59, M S_{\mathrm{e}}=24.01\right.$, $p<.01]$. Each of the four groups classified the objects at high levels, although differences among the four groups did differ significantly $\left[F(3,79)=2.93, M S_{\mathrm{e}}=31.75\right.$, $p<.05]$. This main effect arose because the sighted-only group (.941) performed better than the other three groups $(.87-.89 ; p<.05$ in each case), with the three groups not differing from each other $(p>.05$; LSD post hoc test). The object type (prototype, old, new) $\times$ group interaction was not significant $[F(6,158)=1.81, p=.10]$.

The bottom panel of Figure 5 shows the likelihood that the prototype, old, and new objects were called "old." These rates differed substantially among the three types of object types, with the rates being highest for the training objects (.813), the lowest for the new objects (.190), and intermediate rates for the prototype (.742) $\left[F(2,158)=240.38, M S_{\mathrm{e}}=64.08, p<.01\right]$. The main effect of groups was significant $\left[F(3,79)=3.51, M S_{\mathrm{e}}=\right.$ $82.40, p<.05]$, as was the group $\times$ object type interaction $\left[F(6,158)=10.08, M S_{\mathrm{e}}=64.08, p<.01\right]$. Contrary to our expectations, the blind subjects had the highest rates of calling an object "old" (.649), followed by the 
sighted but blindfolded (.582) and the sighted only (.576), with the sighted and touching subjects having the lowest rate (.519). However, as is clear from Figure 5, these overall rates must be qualified by dramatically differing rates of calling the prototype "old." These rates ranged from a low of .543 (ST), to .700 (S-BF), to .725 (S), to 1.000 (B). These differences are all the more striking given that the blind subjects had the lowest false alarm rates of any group for the new objects (.115), with a high of .315 for the S-BF groups.
An inspection of the individual transfer stimuli for each of the four groups is shown in Figure 6. The top panel shows the likelihood of calling each transfer object "old" for the two groups that relied exclusively on touch alone, the S-BF and B group subjects; the bottom panel shows the performance for the $\mathrm{S}$ and $\mathrm{ST}$ groups. In each figure, Stimuli 1-8 are the old (training) objects, 9-18 are the new objects, and the two prototypes are 19 and 20.

Comparison of the blind subjects with the S-BF groups showed that the S-BF group fared worse than the blind on
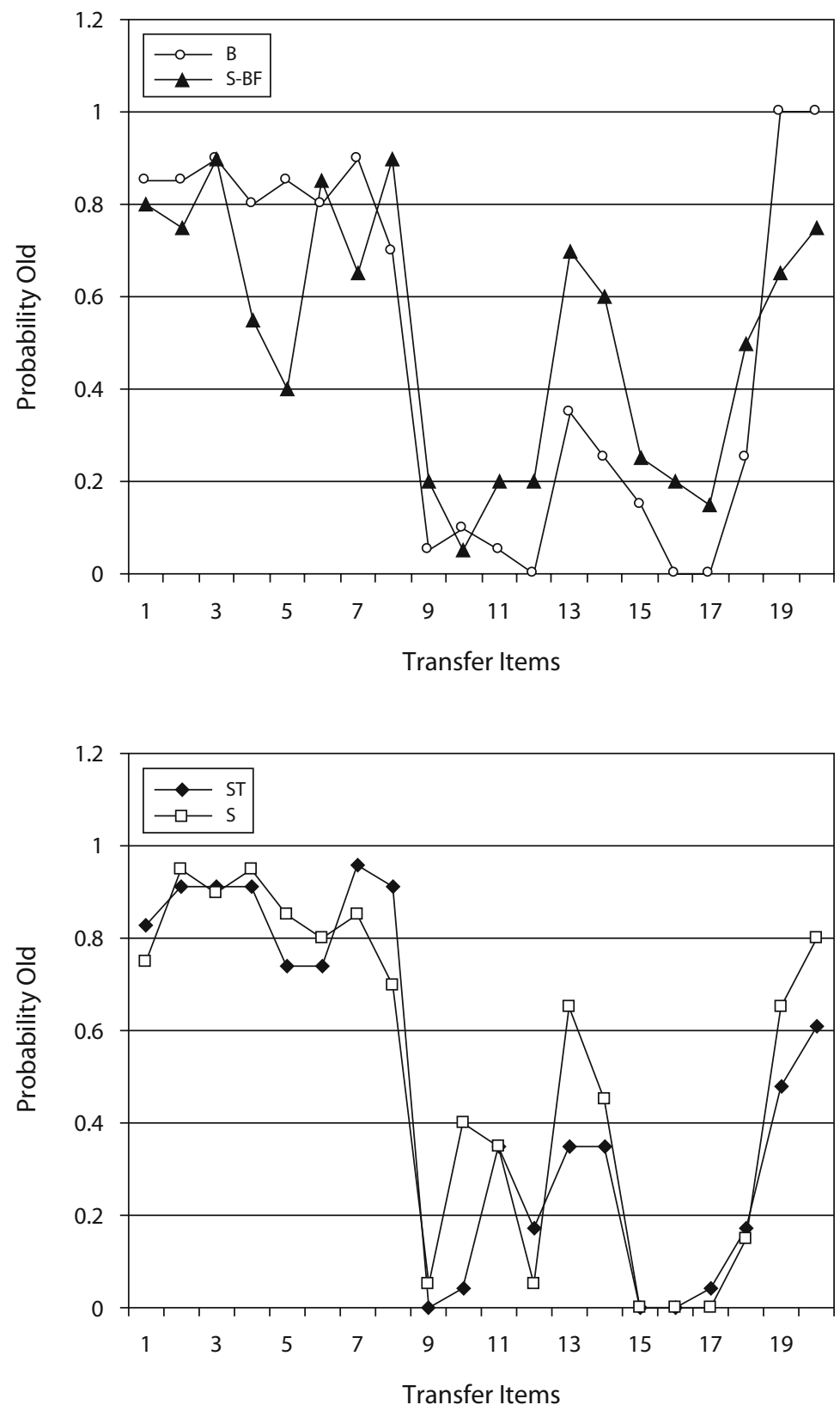

Figure 6. The probability of calling the transfer stimuli "old." Stimuli 1-8 are the training stimuli, 9-18 are the new stimuli, and the prototypes are Stimuli 19 and 20. The top panel shows the blind and sighted but blindfolded subjects; the bottom panel shows the sighted-only and the sighted and touching subjects. B, blind; S-BF, sighted but blindfolded; ST, sighted and touching; S, sighted only. 
the old objects, with 3 of the 8 training objects having a hit rate that ranged from .40 to .65 . In contrast, only 1 of the 8 training objects for the blind subjects had a hit rate below .80. Similarly, the S-BF subjects called the new objects "old" at a rate higher than the blind subjects for 9 of the 10 objects, with 3 of these objects receiving a false alarm rate between .50 and .70 (excluding the prototypes). Only 2 of the 10 new objects were false alarmed at a rate that exceeded .20 by the blind subjects, with these being at a relatively low rate of .20-.35. It is worth noting that the high rates of false alarms by the blind to the category prototypes cannot be due to the particular shape of the prototype (Shape 2 for Category A and Shape 4 for Category B), because new objects that had the same shape as the prototype (Objects 9, 12, 17, 18) had false alarm rates that averaged less than $10 \%$.

A comparison of the ST and S subjects revealed that the ST subjects were slightly, but consistently, more accurate than the S subjects in calling the old objects "old" and the new objects "new."

Given the divergent rates of calling objects "old" for the different groups, a signal detection analysis (Green \& Swets, 1966) was done, the results of which are shown in Figure 7.

This analysis revealed a couple of interesting results. First, the blind subjects demonstrated the greatest $d^{\prime}$ separation among the three types of objects-old, new, and prototype - and the sighted but blindfolded subjects showed the least separation. This suggests that the blind subjects discriminated these three object types to the greatest extent. Second, the blind subjects were the only ones to have higher oldness ratings for the category prototype than for the old training objects - every other group

\section{Signal Detection Measures}

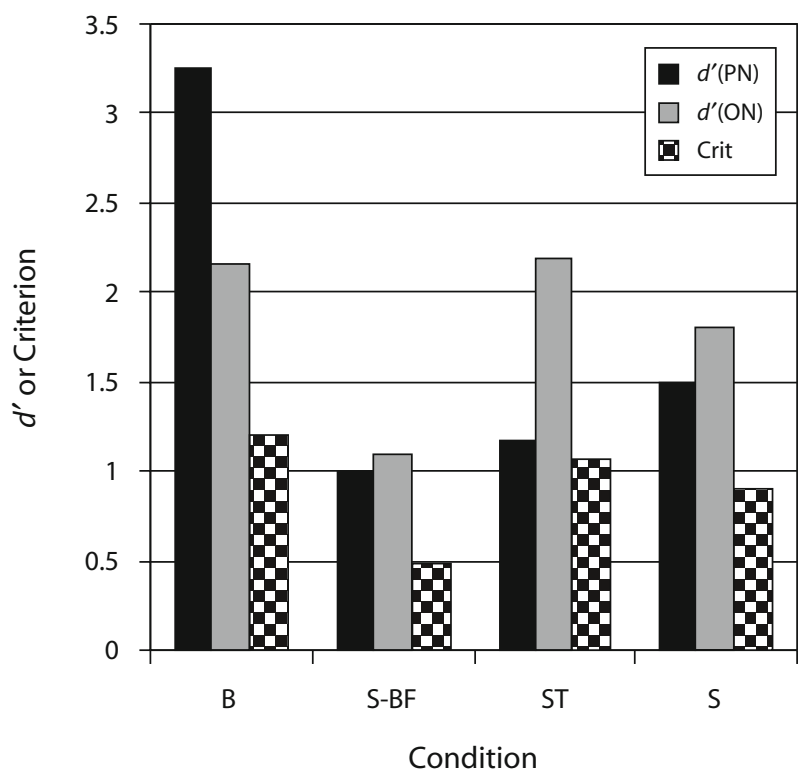

Figure 7. Signal detection measures on the recognition transfer test for each of the four groups (B, blind; S-BF, sighted but blindfolded; ST, sighted and touching; S, sighted only). revealed higher oldness ratings for the old objects, with the greatest $d^{\prime}$ separation between the old and prototype objects in the group that both viewed and touched the objects. Finally, the blind subjects had a criterion placement that excluded, from oldness ratings, the highest proportion of new objects from being mistakenly called "old." In that sense, the blind subjects might be considered to be the most conservative responders of the four groups. However, this conservative placement of the criterion did not preclude these subjects from always making false alarms to one particular object within each category - the category prototype. ${ }^{4}$

Finally, all analyses were redone on the blind subjects who had lost their vision early ( $0-3$ years), intermediate (4-9 years), or late ( $>12$ years) in age. None of these analyses - learning, classification or recognition on the transfer test, or recognition performance across the full set of 20 objects - revealed either a main effect of age of blindness or any interactions with type of transfer object. In fact, the within-subjects variance, when computed for each group and for each of the four tests, was smallest for the blind subjects, greatest for the blindfolded group, and intermediate for the sighted-only and sighted and touching subjects. In effect, the blind subjects functioned more alike in learning, recognition, and classification, in spite of their differing age of blindness, than did the subjects in any of the remaining groups. ${ }^{5}$

\section{DISCUSSION}

The present study revealed a number of provocative, albeit preliminary, findings about haptic concepts learned by blind and sighted subjects. First, the blind subjects readily learned categories that were structured so that the forms were linearly separable only if two or more dimensions were used. In fact, the blind subjects appeared to learn the category structure somewhat more rapidly than sighted but blindfolded controls, but no faster than sighted subjects who were either allowed to see and touch the forms in learning or only see these forms. As an aside, the rapid learning by all four groups was unexpected, given the reported difficulty of learning similar categorical structures (e.g., Smith \& Minda, 2000). One obvious possibility - that haptic information is readily apprehended by all subjects - cannot be correct, since the sighted controls who were prohibited from handling the forms also learned rapidly. A reasonable explanation is that the study/test format used in the present study accelerates learning more rapidly than the more traditional learning format of providing feedback only following the subject's assignment of a stimulus into a category. Having subjects learn categories in which the training stimuli could take on any of five values, rather than binary values, should logically make the task more difficult rather than easier. It is possible, of course, that 3-D objects can be learned faster than 2-D objects. A followup study will investigate the role of spatial structure and dimensionality in learning visuohaptic categories.

The most dramatic difference among the four groups occurred on the recognition transfer test. The blind subjects, as a group, differed markedly from the other three 
groups, being least likely to make false alarms to other new forms but also having the highest likelihood of doing so to the category prototype. This outcome supports, to a limited degree, the informal comments by blind subjects that they are required to function more conservatively on a recognition test. However, the false alarms to the category prototype - virtually every blind subject called the category prototype for each category a familiar object - run contrary to this anecdotal view of conservative responding. Their performance contrasted sharply with the other conditions, in which the prototype was called "old" at rates varying between $50 \%$ and $75 \%$. How to interpret the recognition results of the blind subjects, given their excellent generalization performance to the new and prototype patterns on the classification judgment, is problematic. One possibility is that the blind subjects, with their heavy reliance on haptic processing, were most likely to abstract a haptic category prototype from the training stimuli, which resulted in both $100 \%$ accurate classification of the category prototype as well as a false alarm rate of $100 \%$. High rates of classification and false alarms to the category prototype have been reported in numerous studies (e.g., Homa, Smith, Macak, Johovich, \& Osorio, 2001; Metcalfe \& Fisher, 1986; Solso \& Raynis, 1979) and are readily predicted by some models (e.g., Knapp \& Anderson, 1984). This conclusion should be treated with some caution, however, since false recognition of the category prototype is partially determined by the categorical structure: When the prototype is composed of features that exactly match those found in the training patterns (as was the case in the present study), then false recognition rates for the prototype are high and increase further with additional learning trials. When the prototype is constructed from novel feature values, based on the mean value of features contained in the learning set, then false recognition of the category prototype is reduced, especially following multiple learning trials (Homa et al., 2001). Thus, the high rates of false alarming to the category prototype, especially by the blind subjects, should not be taken as strong support for an abstraction mechanism until converging support (e.g., formal models, manipulations of old-new similarity, and prototype distortions) has been obtained.

Two additional results involving the blind subjects were noteworthy. First, age of blindness for our subjects did not have a significant impact on cognitive functioning, either in learning or on the transfer task. Recent studies have demonstrated that early and late blind subjects often function similarly in tasks such as sound localization (Voss et al., 2004) and navigation (Fortin et al., 2008), even though differences in cortical functioning are often revealed in tasks such as Braille reading (Burton, 2003). Second, we were surprised to find that within-subjects variance on all tasks was generally smallest in our blind group, especially in the recognition task that generated the most variance among all subjects, not just the blind. These results would be consistent with the view that severe sensory deprivation results in cortical reorganization (Burton, 2003) that begins rapidly once sight is lost, with age of blindness having a minimal effect, at least for the task used in the present study.

Finally, false alarms to the category prototype were lowest for the group that was allowed to both touch and visually inspect the forms, in both learning and transfer. Inputs from multiple modalities, rather than just one, might help to ensure against false alarming, even to the category prototype. It is noteworthy that these subjects did classify the category prototype at nearly $100 \%$ accuracy but made false alarms to the category prototype only $50 \%$ of the time. In the future, assessing the representation of the stored traces for multiple sensory input concepts might be clarified by restricting transfer to one modality-either touch or vision, but not both - to determine whether subjects are integrating the sensory properties across modalities or whether the modalities help to keep these inputs separated. This paradigm might permit a test of an intriguing hypothesis about what it means to abstract, when the training patterns are received from multiple sensory modalities, rather than just the visual one.

\section{AUTHOR NOTE}

We thank Peter Killeen and Dr. Walter Corteso for their comments on the manuscript. Correspondence relating to this article can be addressed to D. Homa, Department of Psychology, Arizona State University, Tempe, AZ 85287 (e-mail: donhoma@asu.edu).

\section{Note-Accepted by the previous editorial team, when Thomas H. Carr was Editor.}

\section{REFERENCES}

Aiken, E. G. (1969). Auditory discrimination learning: Prototype storage and distinctive features detection mechanisms. Perception \& Psychophysics, 6, 95-96.

BurTon, H. (2003). Visual cortex activity in early and late blind people. Journal of Neuroscience, 23, 4005-4011.

Burton, H., Snyder, A. Z., Diamond, J. B., \& Raichle, M. E. (2002). Adaptive changes in early and late blind: A fMRI study of verb generation to heard nouns. Journal of Neurophysiology, 88, 3359-3371.

Fortin, M., Voss, P., Lord, C., Lassonde, M., Pruessner, J., SaintAMOur, D., ET AL. (2008). Wayfinding in the blind: Larger hippocampal volume and supranormal spatial navigation. Brain, 131, 2995-3005

Green, D. M., \& Swets, J. (1966). Signal detection theory and psychophysics. New York: Wiley.

Homa, D., Goldhardt, B., Burruel-Homa, L., \& Smith, J. C. (1993). Influence of manipulated category knowledge on prototype classification and recognition. Memory \& Cognition, 21, 529-538.

Homa, D., Smith, C., Macak, C., Johovich, J., \& Osorio, D. (2001). Recognition of facial prototypes: The importance of categorical structure and degree of learning. Journal of Memory \& Language, 44, 443-474.

Klatzky, R. L., Lederman, S. J., \& Metzger, V. A. (1985). Identifying objects by touch: An "expert system." Perception \& Psychophysics, 37, 299-302.

KNAPP, A. G., \& ANDERson, J. A. (1984). Theory of categorization based on distributed memory storage. Journal of Experimental Psychology: Learning, Memory, \& Cognition, 10, 616-637.

Lockman, J. J., \& Wright, M. H. (1988, April). A longitudinal study of banging. Paper presented at the International Conference on Infant Studies, Washington, DC.

Medin, D. L., \& Schaffer, M. M. (1978). Context theory of classification learning. Psychological Review, 85, 207-238.

Metcalfe, J., \& Fisher, R. P. (1986). The relation between recognition memory and classification learning. Memory \& Cognition, 14, 164-173. 
Morrongiello, B. A., Humphrey, G. K., Timney, B., Choi, J., \& RoccA, P. T. (1994). Tactual object exploration and recognition in blind and sighted children. Perception, 23, 833-848.

Posner, M. I., \& Keele, S. W. (1968). On the genesis of abstract ideas. Journal of Experimental Psychology, 77, 353-363.

Rosser, E. M. (1967). Categorization and discrimination of tone sequences. Unpublished doctoral dissertation, Harvard University, Cambridge, MA.

RufF, H. A. (1984). Infants' manipulative exploration of objects: Effects of age and object characteristics. Developmental Psychology, 20, 9-20.

Shimizu, Y., SAIDA, S., \& Shimura, H. (1993). Tactile pattern recognition by graphic display: Importance of 3-D information for haptic perception of familiar objects. Perception \& Psychophysics, 53, 43-48.

Smith, J. D., \& MindA, J. P. (2000). Thirty categorization results in search of a model. Journal of Experimental Psychology: Learning, Memory, \& Cognition, 26, 3-27.

Solso, R. L., \& RAYNis, S. A. (1979). Prototype formation of imaged, kinesthetically and visually presented geometric forms. Journal of Experimental Psychology: Human Perception \& Performance, 5, 701-712.

Van Boven, R. W., Hamilton, R. H., Kauffman, T., Keenan, J. D., \& Pascual-Leone, A. (2000). Tactile resolution in blind readers. Neurology, 54, 2230-2236.

Voss, P., Lassonde, M., Gougoux, F., Fortin, M., Guillemot, J. P., \& LEPORE, F. (2004). Early- and late-onset blind individuals show supra-normal auditory abilities in far-space. Current Biology, 14, 1734-1738.

\section{NOTES}

1. Size and texture can be unambiguously ordered (small to large; smooth to rough), but not shape. However, the categories in the present study are linearly separable in two dimensions, and whatever ordering is used for shape does not alter this fact. The ordering for shape shown in
Figure 1 is not totally arbitrary, however, because the five basic shapes can be ordered by the minimum number of cubes needed to be added/ deleted to reproduce another shape.

2. The designation of early and late blindness is variably defined in the literature, with early blind defined as the loss of sight before the age of 3 (Burton, Snyder, Diamond, \& Raichle, 2002), age of 5 (Fortin et al., 2008), or age of 6 (Burton, 2003); late blindness is generally considered to occur in these studies after the age of 10 . Our decision to separate subjects into early, mid-, and late blind is somewhat arbitrary but not inconsistent with the literature. We report analyses for the blind group as a whole as well as by this division.

3 . Two of the five new forms of each category could not be assigned to a category based on the size and texture dimensions alone (Forms 12 and 14 of Category A and 16 and 20 of Category B). However, each of these forms matched one or two of the learning forms of its category and none of the forms in the contrasting category.

4. Our efforts to formally model the transfer results according to current exemplar and prototype models of classification and recognition met with limited success. A major obstacle was how to compute summed similarity across the dimensions of texture, size, and shape. Although we were able to generate reasonable predictions for which stimuli were called "old" and "new," we could not account for the extreme hit rates for the training patterns and the low false alarm rates for the transfer patterns, except for the category prototypes. Our analyses and the full data sets are available upon request.

5. Analyses for the blind subjects were redone with the 2 subjects who could read large print removed. None of these analyzes altered statistical outcomes for learning, classification, or recognition. In fact, the performance for these 2 subjects was indistinguishable from the other blind subjects on these tasks. These 2 subjects, who were blindfolded during the task, could identify edges in bright contrast but could not otherwise navigate from vision alone.

(Manuscript received May 4, 2007; revision accepted for publication December 5, 2008.) 